\title{
A General Framework for Ordering Fuzzy Sets
}

\author{
Ulrich Bodenhofer \\ Software Competence Center Hagenberg, A-4232 Hagenberg, Austria
}

\begin{abstract}
Orderings and rankings of fuzzy sets have turned out to play a fundamental role in various disciplines. Throughout the previous 25 years, a lot a different approaches to this issue have been introduced, ranging from rather simple ones to quite exotic ones. The aim of this paper is to present a new framework for comparing fuzzy sets with respect to a general class of fuzzy orderings. This approach includes several known techniques based on generalizing the crisp linear ordering of real numbers by means of the extension principle, however, in its general form, it is applicable to any fuzzy subsets of any kind of universe for which a fuzzy ordering is known - no matter whether linear or partial.
\end{abstract}

\section{Introduction}

There is no doubt that orderings and rankings are essential in any field related to decision making. Admitting vagueness or impreciseness naturally results in the need for specifying vague preferences in crisp domains, but also in the demand for a framework in which it is even possible to decide between fuzzy alternatives. It is, therefore, not surprising that orderings and rankings of fuzzy sets have become main objects of study in fuzzy decision analysis and related disciplines.

Albeit only scarcely recognized, orderings of fuzzy sets are also important in areas related to fuzzy systems and fuzzy control, where the ordering of a numerical domain is most often used when defining fuzzy sets - there might only be a minority of fuzzy systems or controllers in which expressions like "small", "medium", or "large" do not occur. This ordering, however, is even more crucial as soon as automatic tuning procedures are concerned, which are supposed to give interpretable, i.e. understandable, results [1,5]. Similar questions arise in linguistic approximation $[7,19]$ which may be considered as a kind of inverse procedure - finding a linguistic label for a given fuzzy set. A third application scenario is rule interpolation $[15,16]$ which is concerned with obtaining conclusions for observations which are not covered by any antecedent in a fuzzy rule base. Orderings of fuzzy sets are able to provide criteria for determining between which rules the interpolation should take place [15]. 
Since the 1970s, a host of different methods for ordering or ranking fuzzy sets has been published (see $[6,20-22]$ for detailed reviews). In order to find profound motivations for adding yet another approach, let us review some common characteristics of these methods:

1. As long as linguistic expressions are represented by fuzzy subsets of numerical domains, there is a certain context-dependent notion of indistinguishability. It could be desirable to take this indistinguishability into account, since not only the ranking of alternatives itself, but also the information that the difference between two alternatives is, more or less, negligible could be of interest. All existing methods, however, do not offer the opportunity to integrate indistinguishability which often leads to undesired, counter-intuitive preciseness.

2. All methods are defined for so-called fuzzy quantities - fuzzy subsets of the real numbers. Not only from the theoretical, but also from the practical point of view, it could be interesting to consider arbitrary ordered domains, without any restriction in terms of the underlying domain or linearity of the ordering.

3. The applicability of many ordering methods is restricted to fuzzy quantities having special properties, such as convexity, normality, or continuity (often called fuzzy numbers). The motivation for such restrictions is to guarantee some desirable properties, for example, antisymmetry.

The purpose of this paper is to introduce and investigate an ordering method for arbitrary fuzzy subsets of an arbitrary (fuzzy) ordered domain where indistinguishability is taken into account, too. For proof details and theoretical basics, the reader is referred to [3].

\section{Preliminaries}

In this paper, uppercase letters will be used synonymously for denoting fuzzy sets and their corresponding membership functions. The reader is assumed to be familiar with the basics of triangular norms and their corresponding residual implications $[8,10,14]$. Throughout the whole text, the symbol $T$ is supposed to denote a left-continuous t-norm.

Since, according to the above discussions, the ordering method should be able to cope with vagueness and indistinguishability, all studies in this paper will be based on the similarity-based definition of fuzzy orderings (for extensive studies, see $[3,4,11])$. We only recall the very basic definitions.

Definition 1. A binary fuzzy relation $E$ on a domain $X$ is called fuzzy equivalence relation with respect to $T$, for brevity $T$-equivalence, if and only if the following three axioms are fulfilled for all $x, y, z \in X$ :

(i) Reflexivity: $\quad E(x, x)=1$

(ii) Symmetry: $\quad E(x, y)=E(y, x)$

(iii) $T$-transitivity: $T(E(x, y), E(y, z)) \leq E(x, z)$ 
Definition 2. Let $L: X^{2} \rightarrow[0,1]$ be a $T$-transitive fuzzy relation. $L$ is called fuzzy ordering with respect to $T$ and a $T$-equivalence $E$, for brevity $T$-E-ordering, if and only if it additionally fulfills the following two axioms for all $x, y \in X$ :

(i) $\quad E$-Reflexivity: $\quad E(x, y) \leq L(x, y)$

(ii) $T$-E-antisymmetry: $T(L(x, y), L(y, x)) \leq E(x, y)$

A subclass, which will be of special importance in the following, are socalled direct fuzzifications.

Definition 3. A $T$-E-ordering $L$ is called a direct fuzzification of a crisp ordering $\preceq$ if and only if it admits the following resolution:

$$
L(x, y)= \begin{cases}1 & \text { if } x \preceq y \\ E(x, y) & \text { otherwise }\end{cases}
$$

It is worth to mention that there is a one-to-one correspondence between direct fuzzifications of crisp linear orderings and fuzzy orderings $L$ which additionally fulfill strong completeness [3,4]

$$
\max (L(x, y), L(y, x))=1 .
$$

The modifiers 'at least' and 'at most' with respect to a fuzzy ordering $L$ will be essential for all further investigations. They can be defined by means of hulls/closures.

Definition 4. Suppose that $X$ is supplied with some $T$ - $E$-ordering $L$. Then, for a fuzzy subset $A$ of $X$, the fuzzy sets 'at least $A$ ' and 'at most $A$ ' (with respect to $L$ ), abbreviated $\operatorname{ATL}(A)$ and $\operatorname{ATM}(A)$, respectively, can be defined as follows:

$$
\begin{aligned}
\operatorname{ATL}(A)(x) & =\sup \{T(A(y), L(y, x)) \mid y \in X\} \\
\operatorname{ATM}(A)(x) & =\sup \{T(A(y), L(x, y)) \mid y \in X\}
\end{aligned}
$$

If $L$ coincides with a crisp ordering $\preceq$, we will use the symbols $\operatorname{LTR}(A)$ and $\operatorname{RTL}(A)$ instead (standing for left-to-right and right-to-left continuation, respectively), where one can easily show the following identities:

$$
\begin{aligned}
& \operatorname{LTR}(A)(x)=\sup \{A(y) \mid y \preceq x\} \\
& \operatorname{RTL}(A)(x)=\sup \{A(y) \mid x \preceq y\}
\end{aligned}
$$

In words, $\operatorname{LTR}(A)$ is the smallest superset of a fuzzy set $A$ the membership function of which is non-decreasing with respect to $\preceq$. Correspondingly, for any fuzzy set $A, \operatorname{RTL}(A)$ is the smallest superset whose membership function is non-increasing. In direct analogy, $\operatorname{ATL}(A)$ can be regarded as the smallest superset of $A$ the membership function of which is non-decreasing with respect to the fuzzy ordering $L$; analogously for $\operatorname{ATM}(A)$ (for a detailed and mathematically exact argumentation, see [3]). 
Theorem 1. Provided that a T-E-ordering $L$ is a direct fuzzification of a crisp ordering $\preceq$, the following holds for all fuzzy sets $A$

$$
\begin{aligned}
\operatorname{ATL}(A) & =\operatorname{LTR}(\operatorname{EXT}(A))=\operatorname{ExT}(\operatorname{LTR}(A)), \\
\operatorname{ATM}(A) & =\operatorname{RTL}(\operatorname{EXT}(A))=\operatorname{ExT}(\operatorname{RTL}(A)),
\end{aligned}
$$

where $\operatorname{EXT}(A)$ is the so-called extensional hull of $A$ with respect to $E[13,17]$, which is defined as

$$
\operatorname{EXT}(A)(x)=\sup \{T(A(y), E(y, x)) \mid y \in X\} .
$$

Example 1. Figure 1 shows an simple example demonstrating the actual meaning of the operators ATL and ATM as well as the correspondences of Theorem 1. We consider the following two fuzzy relations on the real numbers:

$$
\begin{aligned}
& E(x, y)=\max (1-|x-y|, 0) \\
& L(x, y)= \begin{cases}1 & \text { if } x \leq y \\
\max (1-x+y, 0) & \text { otherwise }\end{cases}
\end{aligned}
$$

One easily verifies that $E$ is a $T_{\mathbf{L}}$-equivalence and that $L$ is a $T_{\mathbf{L}}$ - $E$-ordering, which directly fuzzifies the natural ordering of real numbers, where $T_{\mathbf{L}}$ stands for the Eukasiewicz t-norm

$$
T_{\mathbf{L}}(x, y)=\max (x+y-1,0) .
$$
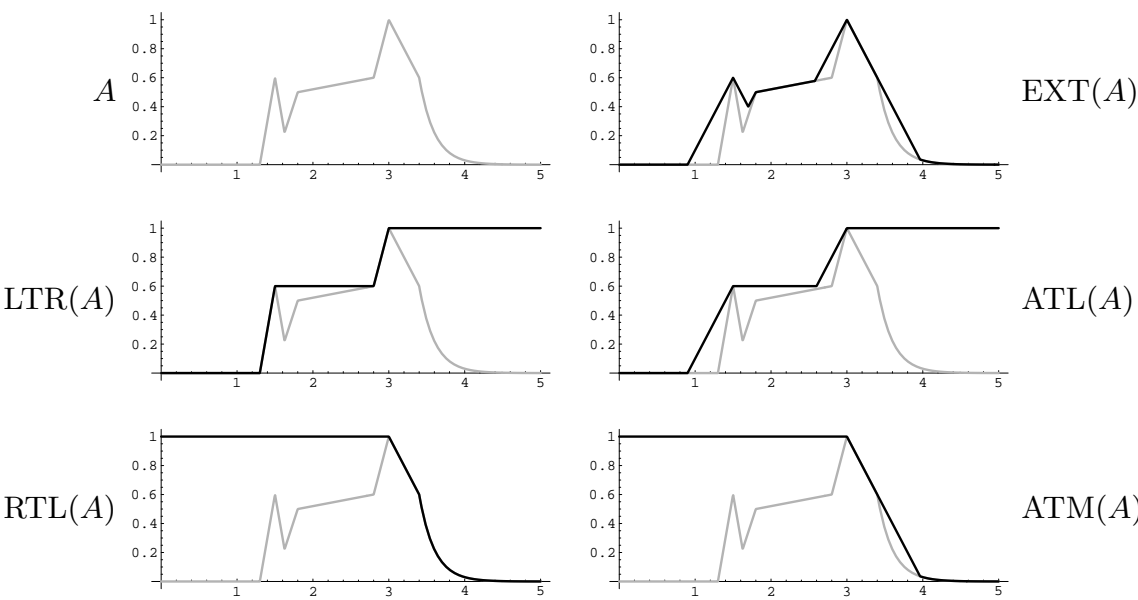

$\operatorname{ATM}(A)$

Fig. 1. A fuzzy quantity $A$ and the results which are obtained when applying various ordering-based operators. 


\section{The Basic Approach}

In order to have a clear motivation, let us start from a well-known ordering procedure for real intervals (with respect to the usual ordering of real numbers):

$$
[a, b] \leq_{I}[c, d] \Longleftrightarrow a \leq c \wedge b \leq d
$$

It is easy to check that $\leq_{I}$ is a partial ordering. The inequality $a \leq c$ means that there are no elements of the set $[c, d]$ which are below the entire interval $[a, b]$. The inequality $b \leq d$, analogously, means that there are no elements of $[a, b]$ which lie completely above $[c, d]$. This criterion can be generalized to arbitrary crisp subsets of an ordered set $(X, \preceq)$ as follows:

$$
M \preceq{ }_{I} N \Longleftrightarrow(\forall x \in N \exists y \in M: y \preceq x) \wedge(\forall x \in M \exists y \in N: x \preceq y)
$$

The following lemma provides an equivalent formulation by means of hulls which will be the basis of all our further generalizations.

Lemma 1. Let a domain $X$ be equipped with an ordering $\preceq$. With the above notations, the following holds for all crisp subsets $M, N \subseteq X$ :

$$
\begin{aligned}
& \operatorname{LTR}(M) \supseteq \operatorname{LTR}(N) \Leftrightarrow \forall x \in N \exists y \in M: y \preceq x \\
& \operatorname{RTL}(M) \subseteq \operatorname{RTL}(N) \Leftrightarrow \forall x \in M \exists y \in N: x \preceq y
\end{aligned}
$$

As an immediate consequence, we obtain that the assertion $M \preceq_{I} N$ is always equivalent to

$$
\operatorname{LTR}(M) \supseteq \operatorname{LTR}(N) \wedge \operatorname{RTL}(M) \subseteq \operatorname{RTL}(N) .
$$

Since the operators LTR and RTL are not restricted to crisp sets, we can write down an extension of $\preceq_{I}$ to fuzzy subsets immediately:

$$
A \preceq_{I} B \Longleftrightarrow(\operatorname{LTR}(A) \supseteq \operatorname{LTR}(B) \wedge \operatorname{RTL}(A) \subseteq \operatorname{RTL}(B))
$$

This means that we are able to order fuzzy sets with respect to a crisp ordering $\preceq$. The generalization to an arbitrary fuzzy ordering is now straightforward.

Definition 5. Let $L$ be a fuzzy ordering on $X$. Then the relation $\preceq$ on $\mathcal{F}(X)$ is defined in the following way:

$$
A \preceq_{L} B \Longleftrightarrow(\operatorname{ATL}(A) \supseteq \operatorname{ATL}(B) \wedge \operatorname{ATM}(A) \subseteq \operatorname{ATM}(B))
$$

Reconsidering Example 1 and Fig. 1, it may be clear that, in a more general setting, the properties $\operatorname{ATL}(A) \supseteq \operatorname{ATL}(B)$ and $\operatorname{LTR}(A) \supseteq \operatorname{LTR}(B)$ correspond to the property that the "left flank" of $A$ is to the left of the "left flank" of $B$. Analogously, $\operatorname{ATM}(A) \subseteq \operatorname{ATM}(B)$ and $\operatorname{RTL}(A) \subseteq \operatorname{RTL}(B)$ relate to the fact that the "right flank" of $A$ is to the left of the "right flank" of $B$. This demonstrates the appropriateness of the approach if we compare these two correspondences with the simple interval order (1). 
Example 2. Figure 2 shows two simple convex fuzzy quantities $A_{1}$ and $B_{1}$ for which naturally $A_{1} \preceq_{I} B_{1}$ (implying $A_{1} \preceq_{L} B_{1}$ for any $L$ directly fuzzifying the natural ordering of real numbers) holds.

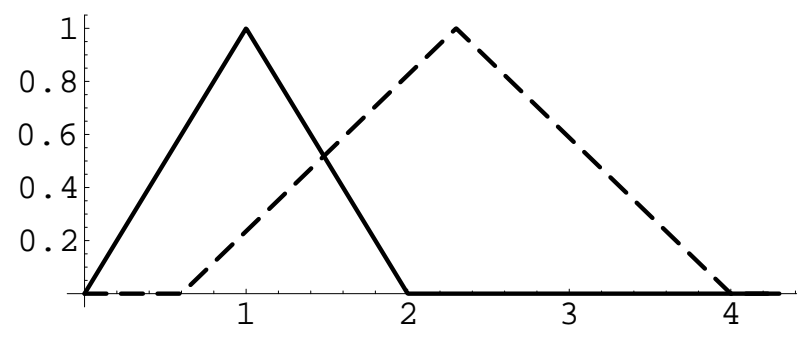

Fig. 2. Two simple convex fuzzy quantities $A_{1}$ (solid line) and $B_{1}$ (dashed line) for which $A_{1} \preceq_{I} B_{1}$ holds.

Correspondingly, Fig. 3 shows two non-convex fuzzy quantities $A_{2}$ and $B_{2}$. In this example, approaches assuming the convexity of the fuzzy sets are not applicable, although it may be obvious from intuition that $A_{2}$ is, in some sense, smaller than $B_{2}$. In the proposed framework, $A_{2} \preceq_{I} B_{2}$ actually holds (again implying $A_{2} \preceq_{L} B_{2}$ for any $L$ directly fuzzifying the natural ordering of real numbers).

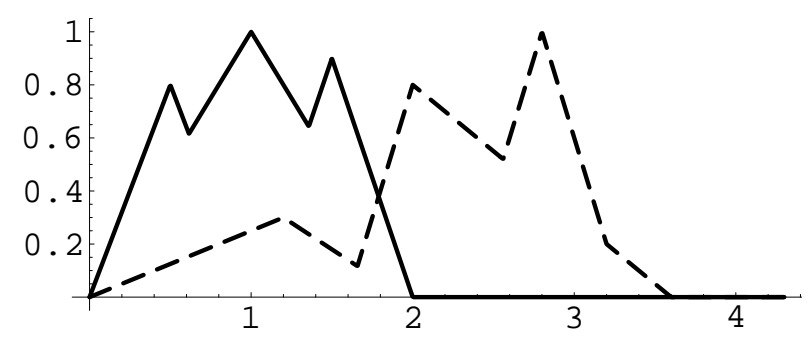

Fig. 3. Two non-convex fuzzy quantities $A_{2}$ (solid line) and $B_{2}$ (dashed line) for which $A_{2} \preceq_{I} B_{2}$ holds.

Remark 1. Note that two fuzzy sets $A$ and $B$ are a priori incomparable if their heights do not coincide, i.e. if

$$
\sup \{A(x) \mid x \in X\} \neq \sup \{B(x) \mid x \in X\} .
$$

For simplicity, therefore, let us assume that the fuzzy sets we deal with are normalized, where we call a fuzzy set $A$ normalized if and only if its height is 1, i.e. $\sup \{A(x) \mid x \in X\}=1$. 


\section{Analysis}

In order to study the properties of relations of type $\preceq_{L}$, let us assume that $L$ is an arbitrary but fixed fuzzy ordering with respect to some t-norm $T$ and a fuzzy equivalence relation $E$.

First of all, it is trivial to see that the relation $\preceq_{L}$ is reflexive and transitive, since it is nothing else than the intersection of two inclusion relations, which are, of course, reflexive and transitive. The next result characterizes antisymmetry, or better, non-antisymmetry in a unique way.

Theorem 2. The following holds for all fuzzy subsets $A, B \in \mathcal{F}(X)$

$$
\left(A \preceq_{L} B \wedge A \succeq_{L} B\right) \Leftrightarrow \operatorname{ECX}(A)=\operatorname{ECX}(B)
$$

where the operator ECX is defined as the minimum intersection of ATL and ATM:

$$
\operatorname{ECX}(A)=\operatorname{ATL}(A) \cap \operatorname{ATM}(A)
$$

Theorem 2, however, only provides a valuable insight if we know more about the inside of the operator ECX. If we go back to crisp orderings for a moment, we will see that the above property corresponds to the equality of the convex hulls. In contrast to vector space-based definitions of convexity $[18,23]$, we will use a general ordering-based definition of convexity, where it is easy to check that, for the real number and their natural ordering, the two definitions are equivalent.

Definition 6. Assume we are given a crisp ordering $\preceq$. Then a fuzzy set $A$ is called convex (with respect to $\preceq$ ) if and only if the following property holds for all $x, y, z \in X$ :

$$
x \preceq y \preceq z \Rightarrow A(y) \geq \min (A(x), A(z))
$$

Proposition 1. Given a crisp ordering $\preceq$ on $X$, the smallest convex superset of a given fuzzy set $A$ - the so-called convex hull of $A$ - is uniquely given as

$$
\operatorname{CVX}(A)=\operatorname{LTR}(A) \cap \operatorname{RTL}(A) .
$$

Almost needless to mention, if $L$ is a crisp ordering, the two operators CVX and ECX coincide.

Hence, we obtain that, for some crisp ordering $\preceq$, the relation $\preceq_{I}$ is unable to distinguish between two fuzzy sets $A$ and $B$ if and only their convex hulls coincide.

In the case that we are dealing with an ordering relation $L$ which is not crisp, this argumentation becomes more subtle. It is intuitively clear that $\operatorname{ECX}(A)$ is always something like a generalized convex hull, but this should be made more precise. If $L$ is a direct fuzzification, a clear answer can be given. 
Theorem 3. Let $L$ be a T-E-ordering which is a direct fuzzification of some crisp ordering $\preceq$. Then the following equalities hold for all $A \in \mathcal{F}(X)$ :

$$
\operatorname{ECX}(A)=\operatorname{CVX}(\operatorname{EXT}(A))=\operatorname{EXT}(\operatorname{CVX}(A))
$$

In words, for direct fuzzifications, the operator ECX can be interpreted as a kind of "extensional convex hull".

In any case, we see that neither $\preceq_{I}$ nor $\preceq_{L}$ is guaranteed to be antisymmetric. However, we have found equivalence relations uniquely describing non-antisymmetry. Of course, we can obtain orderings by factorization with respect to the symmetric kernels of $\preceq_{I}$ and $\preceq_{L}$, respectively. From the above consideration, we know that the symmetric kernels can be represented as

$$
\begin{aligned}
& A \cong_{I} B \Longleftrightarrow \operatorname{CVX}(A)=\operatorname{CVX}(B) \\
& A \cong_{L} B \Longleftrightarrow \operatorname{ECX}(A)=\operatorname{ECX}(B)
\end{aligned}
$$

and we obtain the following result.

Theorem 4. The relation $\preceq_{I}$ is an ordering on $\mathcal{F}(X) / \preceq_{I}$ which is isomorphic to the set of convex fuzzy subsets:

$$
\mathcal{F}_{I}(X)=\{A \in \mathcal{F}(X) \mid A=\operatorname{CVX}(A)\}
$$

Analogously, the relation $\preceq_{L}$ is an ordering on $\mathcal{F}(X) / \cong_{L}$ which is isomorphic to the set of extensional convex fuzzy subsets:

$$
\mathcal{F}_{L}(X)=\{A \in \mathcal{F}(X) \mid A=\operatorname{ECX}(A)\}
$$

The above results have a different quality if we compare them with the existing approaches which restricted to some special classes of fuzzy subsets in advance (e.g. $[12,16])$ just to preserve properties, such as antisymmetry. The new method is not restricted to (extensional) convex fuzzy sets. It can distinguish between any two arbitrary fuzzy subsets as long as their (extensional) convex hulls do not coincide. Since non-antisymmetry is characterized by an equivalence relation, it is possible to define orderings of the equivalence classes in order to obtain an even broader class of fuzzy subsets for which antisymmetry is satisfied [3].

\section{Fuzzification}

If we consider the two convex fuzzy quantities in Fig. 4 , it is easy to see that, if we construct $\preceq_{I}$ by means of the natural ordering of real numbers, these two triangular fuzzy quantities are incomparable. The question is whether it is natural at all to compare vague phenomena crisply or if, as the example in Fig. 4 suggests, this directly leads to artificial preciseness. 


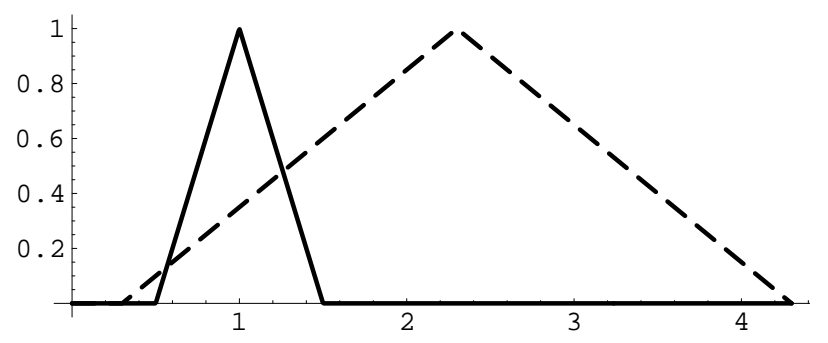

Fig. 4. Two convex fuzzy quantities $A_{3}$ (solid line) and $B_{3}$ (dashed line) which are incomparable.

In this section, we want to overcome this problem by allowing intermediate degrees to which a fuzzy set is smaller or equal than another. For this purpose, let us reconsider the definition of $A \preceq_{L} B$ :

$$
\operatorname{ATL}(A) \supseteq \operatorname{ATL}(B) \wedge \operatorname{ATM}(A) \subseteq \operatorname{ATM}(B)
$$

If we want to make this crisp expression fuzzy, we have to specify (1) a fuzzy concept of subsethood and (2) a conjunction operation.

Definition 7. For a left-continuous t-norm $T$, the residual implication (residuum) is defined as

$$
\vec{T}(x, y)=\sup \{z \in[0,1] \mid T(x, z) \leq y\}
$$

while the corresponding biimplication (equivalence) is defined as

$$
\overleftrightarrow{T}(x, y)=\min (\vec{T}(x, y), \vec{T}(y, x))
$$

In the framework of many-valued predicate logics based on residuated lattices $[9,10]$, it is natural to define the degree of inclusion of a fuzzy set $A$ in another fuzzy set $B$ as $[2,3,8]$

$$
\operatorname{INCL}_{T}(A, B)=\inf _{x \in X} \vec{T}(A(x), B(x))
$$

where the infimum can be considered as a generalization of the universal quantifier.

Theorem 5. The relation $\mathrm{INCL}_{T}$ is a fuzzy ordering on $\mathcal{F}(X)$ with respect to $T$ and the $T$-equivalence

$$
\operatorname{SIM}_{T}(A, B)=\inf _{x \in X} \stackrel{\leftrightarrow}{T}(A(x), B(x)) .
$$

If we replace the Boolean conjunction $\wedge$ by the minimum t-norm and the usual inclusion $\subseteq$ by $\mathrm{INCL}_{T}$, we obtain the following generalization of $\preceq_{L}$ :

$$
\mathcal{L}_{L}(A, B)=\min \left(\operatorname{INCL}_{T}(\operatorname{ATL}(B), \operatorname{ATL}(A)), \operatorname{INCL}_{T}(\operatorname{ATM}(A), \operatorname{ATM}(B))\right)
$$


which is, in fact, a fuzzy ordering of fuzzy sets - perfectly fitting to the results of Theorems 2 and 4:

Theorem 6. The fuzzy relation $\mathcal{L}_{L}$ is fuzzy ordering on $\mathcal{F}(X)$ with respect to $T$ and the fuzzy equivalence relation

$$
\mathcal{E}_{L}(A, B)=\operatorname{SIM}_{T}(\operatorname{ECX}(A), \operatorname{ECX}(B))
$$

So far, it remains an open question in which way the crisp ordering $\lesssim L$ and the fuzzy ordering $\mathcal{L}_{L}$ are related to each other. The next result gives an exhaustive answer:

Theorem 7. The following characterization of the kernel of $\mathcal{L}_{L}$ holds:

$$
\forall A, B \in \mathcal{F}(X):\left(\mathcal{L}_{L}(A, B)=1 \Longleftrightarrow A \preceq_{L} B\right)
$$

The relationship between $\mathcal{E}_{L}$ and $\cong_{L}$ is given analogously:

$$
\forall A, B \in \mathcal{F}(X):\left(\mathcal{E}_{L}(A, B)=1 \Longleftrightarrow A \cong_{L} B\right)
$$

In particular, this entails that $\preceq_{L}$ is a subrelation of $\mathcal{L}_{L}$ which implies that the comparability of two fuzzy sets with respect to $\mathcal{L}_{L}$ cannot be worse than comparability with respect to $\preceq_{L}$. The following example will show that the problem of artificial strictness when comparing fuzzy sets with $\preceq_{L}$ is perfectly solved if they are compared with the relation $\mathcal{L}_{L}$.

Example 3. If we choose $L$ to be the crisp linear ordering of real numbers, i.e. $L=\chi_{\leq}$, and $T=T_{\mathbf{L}}$, Theorem 7 implies the following if we apply $\mathcal{L}_{L}$ to the fuzzy quantities from Example 2:

$$
\begin{aligned}
& \mathcal{L}_{L}\left(A_{1}, B_{1}\right)=1 \\
& \mathcal{L}_{L}\left(A_{2}, B_{2}\right)=1
\end{aligned}
$$

Moreover, it is easy to verify the following equalities:

$$
\begin{aligned}
& \mathcal{L}_{L}\left(B_{1}, A_{1}\right)=0 \\
& \mathcal{L}_{L}\left(B_{2}, A_{2}\right)=0
\end{aligned}
$$

Now let us reconsider the two fuzzy quantities $A_{3}$ and $B_{3}$ shown in Fig. 4. Using the same $L$ and $T$ as above, we obtain

$$
\begin{aligned}
& \mathcal{L}_{L}\left(A_{3}, B_{3}\right)=0.9 \\
& \mathcal{L}_{L}\left(B_{3}, A_{3}\right)=0
\end{aligned}
$$

which seems quite a reasonable result.

Figure 5 shows two fuzzy quantities $A_{4}$ and $B_{4}$ which would be incomparable with respect to $\preceq_{I}$, too. Using the fuzzification we obtain

$$
\begin{aligned}
& \mathcal{L}_{L}\left(A_{4}, B_{4}\right)=\frac{5}{8}=0.625, \\
& \mathcal{L}_{L}\left(B_{4}, A_{4}\right)=\frac{5}{12}=0.41 \dot{6} .
\end{aligned}
$$




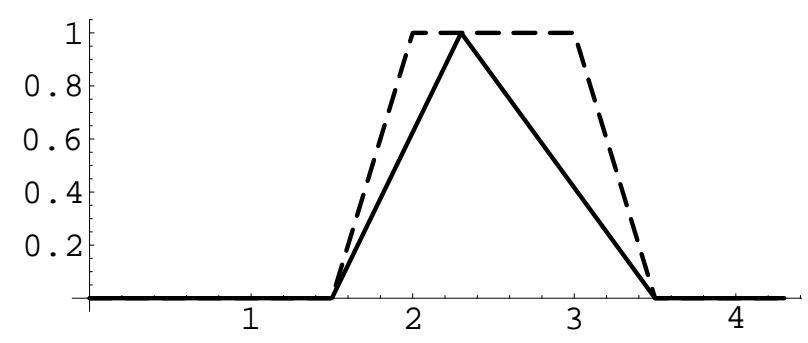

Fig. 5. Two convex fuzzy quantities $A_{4}$ (solid line) and $B_{4}$ (dashed line).

\section{Concluding Remarks}

In this paper, a general method for ordering fuzzy sets with respect to fuzzy orderings was introduced. We have seen that the restriction to certain subclasses of fuzzy sets is not necessary in this approach.

Since it is often not desirable or natural to compare fuzzy sets crisply, a straightforward fuzzification of the ordering approach has been carried out leading to fuzzy orderings of fuzzy sets.

The reader should be aware, as noted in Remark 1, that different heights of two fuzzy sets immediately imply incomparability with respect to $\preceq_{L}$. If the fuzzy variant $\mathcal{L}_{L}$ is taken, this problem is slightly solved, but still in a way which is far from being satisfactory. For a detailed discussion and a possible solution, the reader is referred to [3].

\section{Acknowledgements}

This work has partly been done in the framework of the Kplus Competence Center Program which is funded by the Austrian Government, the Province of Upper Austria and the Chamber of Commerce of Upper Austria, and partly at the Fuzzy Logic Laboratorium Linz-Hagenberg which is part of to the Department of Algebra, Stochastics, and Knowledge-Based Mathematical Systems of the Johannes Kepler University, Linz, Austria.

\section{References}

1. R. Babuška. Construction of fuzzy systems - interplay between precision and transparency. In Proc. ESIT 2000, pages 445-452, Aachen, 2000.

2. W. Bandler and L. Kohout. Fuzzy power sets and fuzzy implication operators. Fuzzy Sets and Systems, 4:183-190, 1980.

3. U. Bodenhofer. A Similarity-Based Generalization of Fuzzy Orderings, volume C 26 of Schriftenreihe der Johannes-Kepler-Universität Linz. Universitätsverlag Rudolf Trauner, 1999. 
4. U. Bodenhofer. A similarity-based generalization of fuzzy orderings preserving the classical axioms. Internat. J. Uncertain. Fuzziness Knowledge-Based Systems, 8(5):593-610, 2000.

5. U. Bodenhofer and P. Bauer. Towards an axiomatic treatment of "interpretability". In Proc. IIZUKA2000, pages 334-339, Iizuka, October 2000.

6. G. Bortolan and R. Degani. A review of some methods for ranking fuzzy subsets. Fuzzy Sets and Systems, 15:1-19, 1985.

7. F. Eshrag and E. H. Mamdani. A general approach to linguistic approximation. Int. J. Man-Mach. Stud., 11:501-519, 1979.

8. S. Gottwald. Fuzzy Sets and Fuzzy Logic. Vieweg, Braunschweig, 1993.

9. S. Gottwald. A Treatise on Many-Valued Logics. Studies in Logic and Computation. Research Studies Press, Baldock, 2001.

10. P. Hájek. Metamathematics of Fuzzy Logic, volume 4 of Trends in Logic. Kluwer Academic Publishers, Dordrecht, 1998.

11. U. Höhle and N. Blanchard. Partial ordering in $L$-underdeterminate sets. Inform. Sci., 35:133-144, 1985.

12. E. E. Kerre, M. Mareš, and R. Mesiar. On the orderings of generated fuzzy quantities. In Proc. IPMU'98, volume 1, pages 250-253, 1998.

13. F. Klawonn and R. Kruse. Equality relations as a basis for fuzzy control. Fuzzy Sets and Systems, 54(2):147-156, 1993.

14. E. P. Klement, R. Mesiar, and E. Pap. Triangular Norms, volume 8 of Trends in Logic. Kluwer Academic Publishers, Dordrecht, 2000.

15. L. T. Kóczy and K. Hirota. Ordering, distance and closeness of fuzzy sets. Fuzzy Sets and Systems, 59(3):281-293, 1993.

16. L. T. Kóczy and K. Hirota. Size reduction by interpolation in fuzzy rule bases. IEEE Trans. Syst. Man Cybern., 27(1):14-25, 1997.

17. R. Kruse, J. Gebhardt, and F. Klawonn. Foundations of Fuzzy Systems. John Wiley \& Sons, New York, 1994.

18. R. Lowen. Convex fuzzy sets. Fuzzy Sets and Systems, 3:291-310, 1980.

19. V. Novák. Fuzzy Sets and Their Applications. Adam-Hilger, Bristol, 1989.

20. X. Wang and E. E. Kerre. On the classification and the dependencies of the ordering methods. In D. Ruan, editor, Fuzzy Logic Foundations and Industrial Applications, International Series in Intelligent Technologies, pages 73-90. Kluwer Academic Publishers, Boston, 1996.

21. X. Wang and E. E. Kerre. Reasonable properties for the ordering of fuzzy quantities (I). Fuzzy Sets and Systems, 118:375-385, 2001.

22. X. Wang and E. E. Kerre. Reasonable properties for the ordering of fuzzy quantities (II). Fuzzy Sets and Systems, 118:387-405, 2001.

23. L. A. Zadeh. Fuzzy sets. Inf. Control, 8:338-353, 1965. 